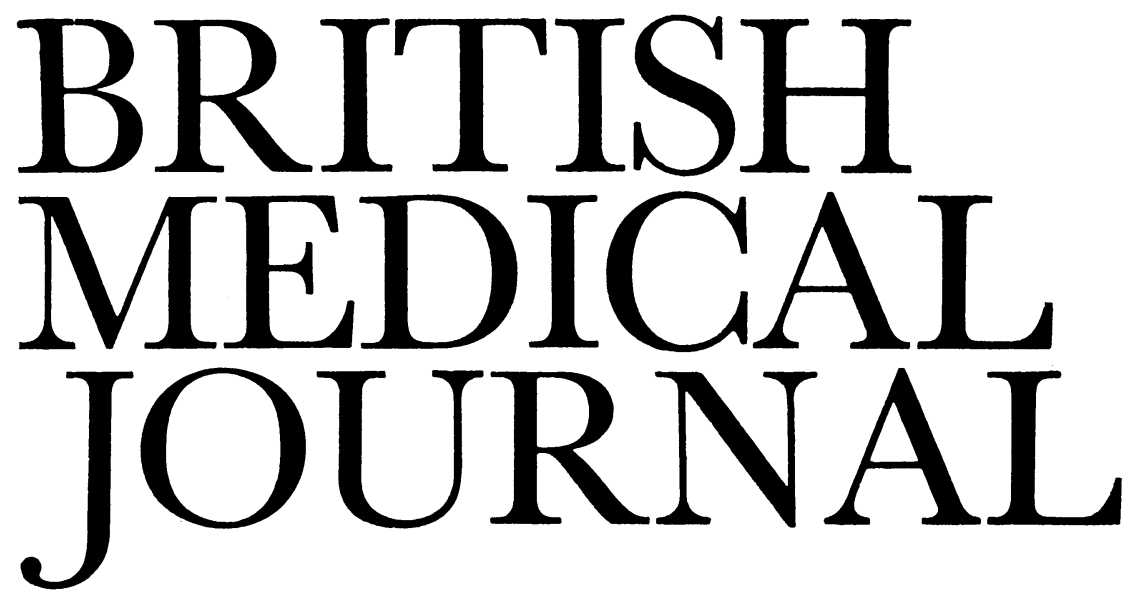

\title{
Plasma sodium concentration: bearer of false prophecies?
}

Occasionally measurement of the serum or plasma concentration of sodium may give rise to falsely low results when carried out on a flame photometer or by an indirect reading ion selective electrode. The patients at risk are those with abnormally high serum concentrations of lipid or protein. These are, however, few: initially all low sodium concentrations as measured by flame photometry should be regarded as genuine.

Measurement of the sodium concentrations of large numbers of specimens of serum or plasma became possible in routine clinical chemistry laboratories some 35 years ago with the advent of the flame photometer. ${ }^{1}$ Before that, measurement of sodium was laborious, time consuming, and imprecise-and the same was true of potassium, measurement of which is equally suited to flame photometry. Indeed this technique had a virtual monopoly until suitable ion selective electrodes became available. ${ }^{2}$

As with many new techniques there are good arguments for and against change, but in this instance the physical principle of measurement differs-a fact which must be understood and taken into account by the clinical chemist. These technicalities do not usually concern or interest the clinician unless they affect the reported result to a substantial extent. Such variation may occur with measurements of serum concentrations of sodium, and both clinicians and clinical chemists need to be aware of the relevant circumstances.

The measurement of sodium by flame photometry determines the number of sodium atoms in a defined unit volume of serum or plasma. The ion selective electrode, by contrast, determines the activity of sodium in the solution rather than its concentration. The concept of activity is one which is familiar to physical chemists but less so to medical scientists and clinicians. It may best be described as a measure of the number of atoms which act truly and wholly as ions in a defined unit volume of electrolytic solution. The difference between concentration and activity is reduced as the solution becomes more dilute. In other words, the difference is less in a plasma sample which has been diluted, say, 1 in 200, before measurement than in the same undiluted sample.

Several factors contribute to the difference between concentration and activity, and these vary in magnitude among different specimens of plasma. The main factor of clinical relevance is the volume occupied by lipid and protein. Because lipids and proteins exclude water the volume they occupy is not available to low molecular weight electrolytes such as sodium and potassium. Measurement by flame photometry does not take this into account, whereas that by ion selective electrodes does. Some ion selective electrode instruments have an inbuilt dilution step which makes their results more similar to those of the flame photometer since there is better agreement between concentration and activity at higher dilution. Others (referred to as direct reading ion selective electrodes) do not, and these generally give a higher value than flame photometry. In other words, the flame photometer measures the amount of electrolyte in the total plasma volume, while the ion selective electrode measures the amount in the plasma water.

For plasma sodium this difference is up to $3 \mathrm{mmol}(\mathrm{mEq}) / 1$ for most samples, with some variation among instruments. Differences of this magnitude are not of clinical importance, but in patients where lipid or protein concentrations or both are raised the difference may be more substantial. For example, in patients with diabetes, familial hyperlipoproteinaemia, myeloma, or those being given intravenous nutrition differences of up to $30 \mathrm{mmol} / 1$ may occur, ${ }^{3}$ and differences of over $100 \mathrm{mmol} / 1$ have been implied. ${ }^{4}$ Similar variations are seen with potassium and all other low molecular weight electrolytes, but the problem is potentially of more clinical importance with sodium than for other electrolytes since its physiological range is relatively smaller.

At first sight the problem would appear to be avoidable if the health care professions agreed not to use direct reading ion selective electrode instruments. It now appears, however, that "true concentration" in plasma water fraction is the correct physiological measurement on which patient management should be based: that is, a direct reading ion selective electrode may give the more "correct" value. The term pseudohyponatraemia has been used to describe the findings when a flame photometer is used and was the subject of a leading article in the Lancet a few years ago $^{5}$ which commented on the potential dangers.

The Scientific Committee of the Association of Clinical Biochemists has been concerned with this problem for some time now, and we believe our clinical colleagues should be alerted to its importance. The time is opportune in view of the recent leading article on "Clinical biochemistry nearer the patient."6 The present tendency is for specialised units such 
as intensive care and cardiac surgery to wish to make their own measurements of electrolytes and to buy direct reading ion selective electrodes, which are convenient to use. Routine clinical chemistry laboratories, however, have continued to purchase flame photometers or indirect reading ion selective electrodes, as these instruments generally have a higher capacity. Inevitably, the discordant results from the two systems will give rise to confusion in interpretation.

The solution to this dilemma may be a compromise whereby laboratories will decide to continue to assay most specimens on high capacity indirect reading instruments but will retain a direct reading ion selective electrode to monitor those few patients with pseudohyponatraemia. Clinicians and clinical chemists must understand the problem so that they can select the correct samples for direct reading ion selective electrode measurement without creating an impossibly large workload for the instrument-and they must also realise that the two different types of instruments will give different answers in a small minority of patients.

H G J WORTH

(On behalf of the Scientific Committee,

Top Grade Biochemist,

King's Mill Hospital,

Sutton-in-Ashfield,

Nottinghamshire NG17 4JL

${ }^{1}$ Hald PM. The flame photometer for the measurement of sodium and potassium in biological materials. F Biol Chem 1947;167:499-510.

2 Friedman SM, Wong S-L, Walton JH. Glass electrode measurements of blood sodium and potassium in man. 7 Appl Physiol 1963;18:950-4.

3 Forrest ARW, Shenkin A. Dangerous pseudohyponatraemia. Lancet $1980 ; \mathrm{ii}: 1256$.

- Frier BM, Steer CR, Baird JD, Bloomfield S. Misleading plasma electrolytes in diabetic children with severe hyperlipidaemia. Arch Dis Child $1980 ; 55: 771-5$.

6 Anonymous. Dangerous pseudohyponatraemia. Lancet 1980;ii:1121.

- Marks V. Clinical biochemistry nearer the patient. Br Med $\mathcal{F} 1983 ; 286$ : 1166-7.

\section{Rotavirus in the home and hospital nursery}

Rotaviruses cause most of the severe diarrhoeal illness in children between the ages of 6-9 months and 2 years. By contrast, rotavirus infection in neonates-even in premature infants-is mild. ${ }^{1-4}$ In this, the human infant differs appreciably from the young farm animal, where the younger the animal the more severe the disease; thus for piglets under a week old there is a high mortality from rotavirus infection. A weaning piglet, however, although it may become ill and lose weight, is much less likely to die of the infection. The same applies to calves and lambs. This discrepancy may be explained by the fact that in man maternal antibodies cross the placenta and may confer protection to the neonate. Calves, pigs, and lambs acquire no such antibody from their mothers and have, to rely on antibody in colostrum to protect them from infection.

Children who become infected with rotavirus while they are in hospital usually recover in about eight days and then go home. Possibly these children excrete rotavirus at a later stage, although, until recently, this has been described only in children who are immunocompromised. A second episode of excretion has recently been described by Dearlove et al. ${ }^{5}$ The second infection may have been caused by a different serotype of rotavirus; but previous accounts of endemic infection by rotaviruses in special care baby units of maternity hospitals have all found that the same strain of virus appeared to be "resident" in the hospital and that the strains which were present in the community were not encountered in the nurseries and special care baby units. ${ }^{3} 4$

Once rotaviruses become established within a hospital nursery they are difficult to eradicate. This is not surprising, since viruses survive for days or even weeks on an impervious surface at high or low humidities (though not so well at intermediate humidities) and at ordinary temperatures. ${ }^{6}$ Viruses are excreted in faeces in enormous numbers $-100 \times 10^{9}$ virus particles/g faeces is not unusual. Thus the most exceptional care is required to prevent their transfer from baby to baby, via nurses' hands or on other contaminated articles. Wiping a surface with disinfectant may reduce the infective count by a millionfold but is of little avail if there are ten thousand million particles.

Experience suggests that the only way of eradicating rotavirus from the hospital nursery is to stop admissions to that particular unit until all the mothers and babies in it have gone home. Once the infected unit is empty, it may be disinfected by spray and formaldehyde fumigation and after 10 days or so the staff, provided they are still healthy, can return to ordinary duty.

Episodes of endemic diarrhoea in maternity hospitals, often going on for months, have so far been described only in major centres where the laboratory is able to detect the causative virus. ${ }^{7}$ This sort of infection may be common but underdiagnosed, because until recently the ability to screen for viral infections has been limited. Now that enzyme-linked immunosorbent assay and other diagnostic tests-for example, reverse passive haemagglutination ${ }^{8}$-are becoming generally available, we may expect that many more examples will be reported.

Rotavirus infection in babies, whether symptomatic or asymptomatic, causes them to lose weight. In developed countries this probably does not matter, since the babies soon make it up again. In poor conditions in the tropics, however, Mata $e t ~ a l^{9}$ showed that repeated episodes of diarrhoea caused young children to lose weight to the extent that they never made it up, so that their final growth was stunted.

Children are great disseminators of infection, and so it is not surprising that Grimwood et al (p 575) find that they are the principal spreaders of rotaviruses within families. Although almost every child has antibodies against rotavirus by the age of $5,{ }^{10}$ infections in adults do occur, especially in old people's homes. In one study ${ }^{11}$ antibody titres to one serotype of rotavirus were found to be highest around the age of 10 , and slowly diminished thereafter, so that in people over 80 they were sometimes undetectable. Outbreaks of infection in geriatric units have been described by several authors. ${ }^{1012} 13$

At least four serotypes of rotaviruses are now recognised, ${ }^{14}$ which fall into two or perhaps three subgroups. ${ }^{15}$ Studies in animals have shown that infection by one rotavirus serotype does not protect against infection by a rotavirus of a different serotype. ${ }^{16}$ Most adults will have had some previous contact with most serotypes of rotavirus and are therefore unlikely to be as susceptible to infection as children. In one study, however; in an isolated tribe of South Américan Indians, ${ }^{17}$ when a rotavirus of a serotype to which none of the tribe had been exposed was introduced, people of all ages developed symptoms. Prevention must be important, a live attenuated vaccine might help, ${ }^{18}{ }^{19}$ but there are many difficulties to be overcome before such a vaccine becomes available.

Consultant Virologist,

T H FleweTt

Regional Virus Laboratory

East Birmingham Hospital,

Birmingham B9 5ST 